MS19-P06

\section{Metalloporphyrinic solid frameworks: catalytic activity}

M. Karmele Urtiaga ${ }^{1}$, Arkaitz Fidalgo ${ }^{1}$, Eder Amayuelas ${ }^{1}$, Begoña Bazán $^{2}$, Gotzone Barandika² ${ }^{3}$, María Isabel Arriortua ${ }^{1}{ }^{2}$

1. Mineralogy and Petrology Department, University of the Basque Country (UPV/EHU), Barrio Sarriena s/n, 48940 Leioa, Spain

2. BCMaterials, Basque Center for Materials, Applications and Nanostructures, UPV/EHU Science Park, Barrio Sarriena s/n, 48940 Leioa, Spain

3. Inorganic Chamistry Department, University of the Basque Country (UPV/EHU), Barrio Sarriena s/n, 48940 Leioa, Spain

email: karmele.urtiaga@ehu.eus

Porphyrin molecules are excellent candidates for bridging ligands in building solid networks because of their rigidity, large physical dimensions, tunable peripheral substituents, and an additional metallation site in the core of the fully conjugated macrocyclic compound. Thus, metalloporphyrin complexes play an important role in natural systems, carrying out essential functions in photosynthesis, oxygen transport, electron carrier and catalytic reactions. These functions are especially interesting if reproduced in the solid state. In fact, metalloporphyrinic solid networks can be extended in the three dimensions of the space through covalent and hydrogen bonds as well as through $\pi$ interactions. As a result, robust, thermally stable, solid coordination frameworks SCFs can be produced. ${ }^{1}$ Porous coordination frameworks are called MOFs (metal-organic frameworks), ${ }^{2}$ and their stability is one of their weakest points, so the reticular chemistry of MOFs can be used to produce stable networks based on metalloporphyrins. ${ }^{3}$ When porous networks are produced, applications like gas adsorption and heterogeneous catalysis are available.

In this context, our research group has a large experience in the synthesis and characterization of porphyrin-based SCFs, with different peripheral substituents such as carboxilates and sulfonates: meso-tetra(4-phenyl)porphyrin (TPP), the meso-tetra(4-carboxyphenyl)porphyrin (TCPP), and the meso-tetraphenyl porphine-tetrasulfonate (TPPS). The work herein presented is focused on the catalytic activity of a family of porphyrinic solid frameworks. The as-produced SCFs have been exhaustively characterized. The catalytic activity has been investigated for some reactions: oxidation of alcohols, aldol and Knoevenagel condensations and onepot cascade reaction for the acetal hydrolysis, followed by a C-C Knoevenagel condensation. In most of the studied compounds, this activity, as heterogeneous catalyst, shows high conversion and TOF (turnover frequencies) values.

\section{Acknowledgements}

This work has been financially supported by the "Ministerio de Economía, Industria y Competitividad" (MAT201676739-R (AEI/FEDER, UE)) and the "Gobierno Vasco" (Basque University System Research Groups, IT-630-13) which is gratefully acknowledge. The technical and human support provided by SGIker (UPV/EHU) is gratefully acknowledged.
References:

1. S. Huh, S.-J. Kim, Y. Kim, (2016) CrystEngComm, 18, 345. 2. Y. Cui, B. Li, H. He, W. Zhou, B. Chen, G. Qian, (2016) Acc. Chem. Res., 49, 483.

3. W. Auwaerter, D. Ecija, F. Klappenberger, J.V. Barth, (2015) Nat. Chem., 7, 105.

Keywords: Metalloporphyrin, MOF, catalysis 